RP300

\title{
MEASUREMENT OF FIBER DIAMETERS BY THE DIFFRACTION METHOD
}

\author{
By H. J. McNicholas and H. J. Curtis
}

\section{ABSTRACT}

The classification of wool fibers into standard grades is based entirely on the average diameter of the fibers. The diffraction of light by a bundle of parallel fibers was employed by Thomas Young in 1824 in a simple ingenious instrument for the rapid direct measurement of average diameter, but no thorough investigation has ever been made of the practical possibilities of this method_in the routine grading of wool.

In the present paper a new construction of Young's instrument (the eriometer) is described, and a critical study is made of the accuracy and adaptability of the instrument in the averaging of a wide range of diameters as distributed in a group of fibers. Sources of error and limitations of the method are discussed.

It is found that the eriometer average is in excellent agreement with comparable data obtained with the microscope. The method affords considerable opportunity for the further development of instruments to include additional features desirable in the study of wool or other textile fibers.

\section{CONTENTS}

I. Introduction

II. Description and theory of the eriometer

III. Calibration and performance.

1. Calibration of the eriometer scale

2. Effect of irregularities in form and arrangement of the fibers - -

3. Dispersion of diameters in wool samples.

4. Precision and accuracy of measurements

IV. Discussion and conclusions

\section{INTRODUCTION}

The average diameter ${ }^{1}$ of wool fibers is a dominant dimensional characteristic of the material immediately affecting its value for manufacturing purposes. In the establishment of the United States standards ${ }^{2}$ for wool and wool top the average diameter of the fibers is the sole basis for the classification. Sets of these standards are in general use in the wool industry; they define the official scale of quality in this country in terms of which the wool grader gives numerical expression to his results.

In present commercial practice the routine grading of wool is done by men of long experience in the industry who by merely handling

1 The term "average diameter" is somewhat loosely applied in this connection, for wool fibers are not always circular in cross section, but may exhibit a considerable degree of irregularity in contour. We employ the term, following customary usage, for the average projected width of the fibers as obtained by microscopic methods or by direct measurement of enlarged images projected on a screen. It is also the quantity observed by direct visual inspection.

2 Oficial Standards of the United States for Grades of Wool and Wool Top, U. S. Department of Agriculture, Service and Regulatory Announcements, No. 100; issued Aug. 3, 1926. 
and observing the material assign it intuitively to its proper grade. 'There is no suitable instrument available for the rapid, convenient, and accurate evaluation of wool on the established basis. Various microscopic and optical projection methods have been employed in the laboratory for measuring the diameter of wool and other textile fibers. Such methods are slow and cumbersome, however, requiring a large number of diameter determinations on individual fibers to obtain a reliable basis for grading.

A simple, rapid optical method for direct measurement of the average diameter of a group of fibers was devised by Thomas Young ${ }^{3}$ in 1824 , but no thorough investigation has ever been made of the possibilities of this method in the routine grading of wool. Young's method is based on the phenomenon of diffraction, which is manifested when a light source of small dimensions is viewed through a bundle of fibers which are arranged approximately parallel to one another. Under these conditions a banded light distribution is seen extending laterally and symmetrically on opposite sides of the source in directions perpendicular to the axes of the fibers. From the theory of diffraction a known relation exists between the separation of the bands and the average diameter of the fibers. Young called his instrument the criometer, from its utility in measuring the fibers of wool. He recommended its use for agricultural purposes and indicated how a toiorable approximation to the value of wool might be obtained from its readings. Young also applied his method to the study of other materials, measuring the average size of particles in milk, blood, barley smut, and dust.

Several instruments embodying the principle of this method have been applied since with moderate success by Pijper, ${ }^{4}$ Bergansius, ${ }^{5}$ Millar, ${ }^{6}$ and Emmons ${ }^{7}$ in the measurement of the average size of blood cells under different pathological conditions. Young's method and instrument are again brought to the attention of the textile industry in a recent article by Ewles, ${ }^{8}$ but no experimental data are given on the performance of the instrument.

In the present paper a new design of the eviometer is described. 'The theory of the method is fully explained and some theoretical and experimental data are included on the accuracy of the instrument and on its ability to collect into a single averaged result the effect of a wide variation in projected diameter's as distributed in a group of fibers. Some advantages and disadvantages of the method are considered in rolation to its further development and application.

It is beyond the scope of the present paper to discuss general aspects of the problem of wool classification or the qualifications of diffrent measures which have been suggested as a basis for grading. In a more complete evaluation of wool quality by scientific measurements ${ }^{D}$ various physical and chemical attributes of the fiber must undoubtedly be talien into consideration. No single property of the fiber alone may be expected to yield an accurate index to the value of all types of wool for difierent trade purposes. One important factor

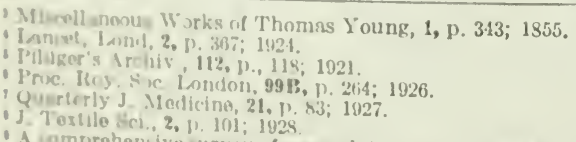

S. tomber, lgeg. 
B. S. Journal of Research, RP300

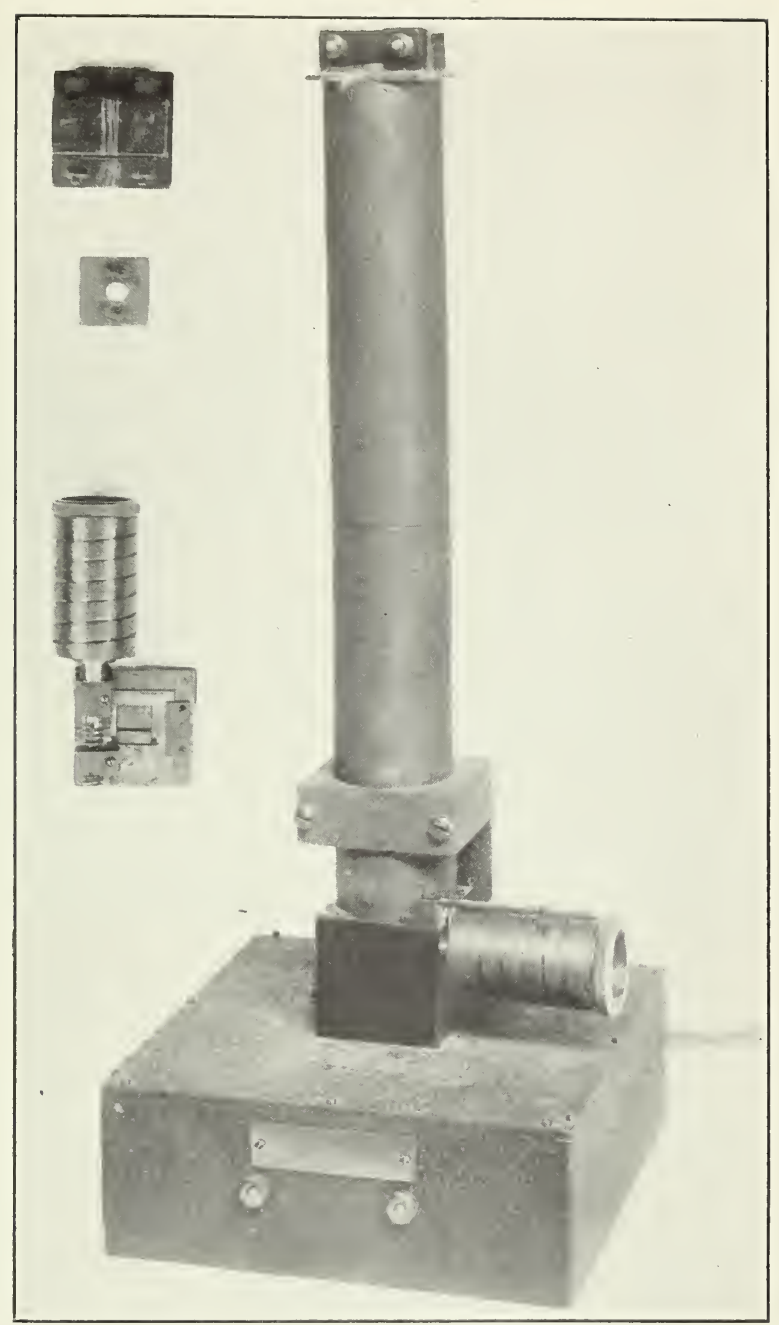

FIgURe 1.-Photograph of the eriometer, with views showing the construction of the slit and the mounting of the fibers 
may be mentioned in this connection because of its general bearing on the subject at band.

It is well known that all dimensional characteristics of the fibers, such as diameter, staple length, crimpiness, cross-sectional area, and contour, each exhibit a wide range of variation within any sample of the material. Our object for measurement is not a single quantity invariant throughout a given sample but is always a wide range of values occurring with variable frequency distribution from sample to sample. If it so happens that this distribution of values for a given property shows no definite central tendency, or concentration, about a single point on the scale of values, then it is evident that no single value, such as the average, can adequately represent the distribution. By itself, the average may have little significance unless supplemented by a measure of variation. A consignment of wool with a high degree of variability and irregularity in certain dimensional properties may not be as suitable for some manufacturing purposes as another consignment with more nearly uniform properties, although both may average the same. The work of Potsdamer and Schenker ${ }^{10}$ indicates that a more reliable and significant procedure for grading on the diameter basis is by comparison of the diameter distribution of the unknown sample with known distributions represented by the set of standards.

In the development of methods and instruments for measuring dimensional properties of the fibers it is therefore of considerable importance to provide, if possible, for rapid and easy determinations of distribution. With the microscope a laborious diameter distribution analysis must precede the determination of the average. It will be shown in the present paper that the diffraction method with the particular instrument herein described provides a rapid direct measure of the arithmetical average along with a definite indication of variation. With further development of instruments based on this method it appears possible to obtain in addition a quantitative expression for the variation.

\section{DESCRIPTION AND THEORY OF THE ERIOMETER}

A photograph of the eriometer is reproduced in Figure 1 with views illustrating the mounting of the fibers and other items of the construction. The design and operation of the instrument will be explained with reference to the diagrams in Figure 2.

At the lower end of an upright observation tube is a rectangular slit $S 1$ approximately $1 \mathrm{~mm}$ wide and $1 \tilde{\mathrm{mm}}$ long. This slit is illuminated through a ground glass diffusion screen by four 10-watt Mazda lamps mounted in a white-lined box which forms the base of the instrument. The narrow illuminated slit serves as a uniform linesource of light for the observation of the diffraction phenomena.

At the top of the tube is a circular aperture $6 \mathrm{~mm}$ in diameter over which a group of fibers may be mounted and arranged approxirnately parallel to the siit. To assist in this adjustment the sample holder may be rotated about the axis of the observation tube.

Looking through the fibers at the illuminated slit one sees then a series of bright diffraction bands separated by narrow darker intervals.

10 The Melliand, 1, pp. 1665, 1776; 1930. 
These bands are arranged parallel to the slit and are extended laterally and symmetrically on both sides. It will be shown presentiy that the spread of these bands, or more specifically, the distance from the center of the bright slit to the first dark band on either side, is- to a close approximation - inversely proportional to the average diameter of the fibers.

For the purpose of measuring this distance the slit is actually made up in three parts, as shown in Figure 1. By means of a screw of known pitch the short outer parts of the slit (approximately $1 \mathrm{~mm}$ in length) may be displaced, simultaneously and laterally, relative to the central stationary part, until they are lined up with the center of the first dark band. This displacement, or its reciprocal value, is then read directly

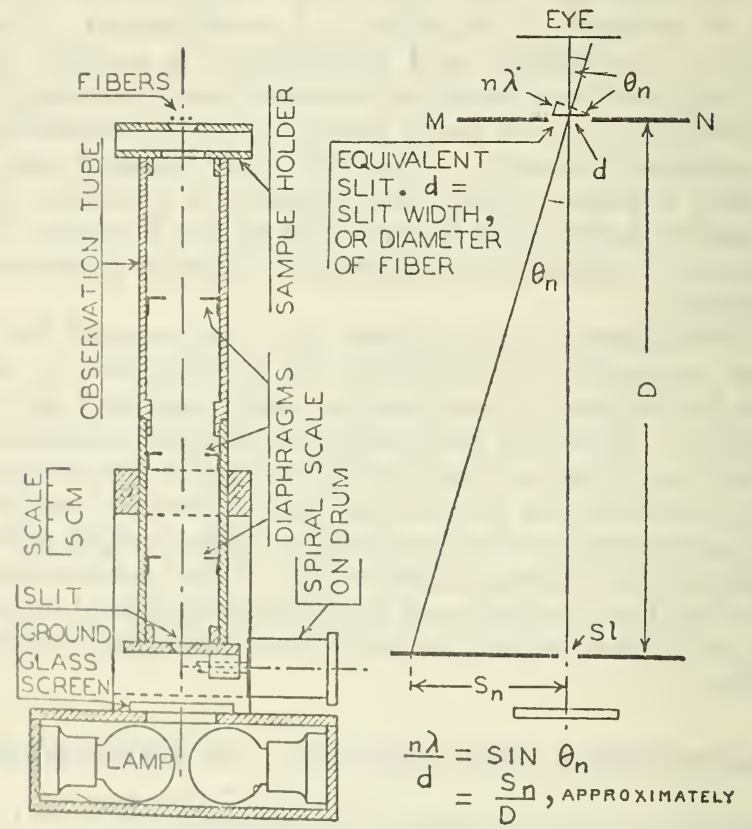

Figune 2.-Diagram of criometer

from $a$ spiral scale on the drum. The use of the ground glass diffusion screen provides for the uniform illumination of the displaced as well as the stationary portions of the slit.

Diaphragnes are inserted in the observation tube to avoid oblique reflection of light from the walls of the tube to the eye, so that the
diffraction bands are thereby observed on a uniformly dark field of
view.

The formation of the bands may be explained as follows by app)lication of well-known optical principles. We consider first the diffraction effects for a single straight fiber of uniform diameter, or for uny small alement of fiber length in a large group of fibers.

In Figure 2 let $S l$ represent a cross scetion of the slit-source and d the diameter of a fiber clement. The length axes of the slit and fior the parallel and the perpendicular distance between them is $D$. For the purpose of this exposition we need consider only the dif- 
fraction effects in a plane which is perpendicular to the fiber element and intersects both this element and the slit. Let this plane be the plane of the paper.

Light from the slit is partially obstructed by the fiber giving rise to diffraction and interference phenomena. By Babinet's principle ${ }^{11}$ the effects observed will be exactly the same if the arrangement is inverted by replacing the fiber with a slit in an opaque screen $M N$, the slit having exactly the same width as the fiber. ${ }^{12}$ In certain directions $\theta_{n}$ from the equivalent slit there will be destructive interference of all secondary wave trains originating at different points across the slit. ${ }^{13}$ These directions are given (fig. 2) by $\sin \theta_{n}=n \lambda / d$, where $n=1,2,3$, etc., is the order of the diffraction band and $\lambda$ is the wave length of light. In directions with intermediate values of $\theta$ reinforcement of secondary wave trains will occur. We may assume for the moment that the source of light is strictly homogeneous with respect to wave length.

The undeviated light passing the equivalent slit, or fiber, is received by the lens of the eye and brought to a focus on the retina, forming a real image of the source-the illuminated slit, Sl. The diffracted light is brought to a focus in like manner as a series of maxima and minima of illumination. This retinal pattern is projected outward, or seen, in the plane of $S^{t}$. The center of the first dark band, for $n=1$, will appear at a distance $s_{1}$ from the center of the slit. The angle $\theta_{1}$ is always so small that the sine may be replaced by the tangent, giving

$$
d=\frac{\lambda D}{s_{1}}=\frac{C}{s_{1}}
$$

where $C=\lambda D$ is the constant of the instrument.

In a group of fibers, all members approximately parallel to the slit, different fiber elements will generally vary in diameter and will simultaneously intercept different portions of the light beam entering the eye. Applying Babinet's principle again, we invert this arrangement, replacing the fibers by a group of equivalent slits in the opaque screen $M N$ (fig. 2). The length axes of these slits are only approximately parallel to one another and are not uniformly spaced in the plane $M N$, so that there is no precise geometrical relationship between all elements of slit length in the group; consequently, there can be no definite phase relations between secondary wave trains originating at different slit elements and propagated in any given direction; no optical effects are observed, therefore, which may be ascribed to the mutual interference of wave trains from different slit elements. We may regard each slit element, or fiber element, as acting independently of all other elements to form its own diffraction pattern of the source, and equation (1) may be applied separately for each element.

11 Wood, Phys. Optics, 2d ed., p. 238.

12 This is analogous to the principle of the optical diffraction grating in which spectra of the same character and in the same position are observed no matter whether the grating consists of black lines on a white ground or white lines on a black ground.

13 The initial phases of the light vibrations mas be regarded as the same at all points in the plane of the equivalent slit. Let a direction of propagation $\theta_{n}$ (fig. 2) be chosen such that the phase difference between secondary wave trains originating, respectively, at the center and either edge of the slit is a whole number $n$ of half-wave lengths. Then the slit width may be subdivided into $2 n$ equal parts and pairs of wave trains from corresponding points in adjacent subdivisions, points always separated by the width of a subdivision, will differ in phase by one-half wave length and consequently will mutually destroy each other. Hence, the resultant intensity of propagation in the chosen direction is zero. There will always be a pair of directions, positive and negative, respectively, for which the condition is fulfilled for any given value of $n$. 
We see that the diffraction pattern of each fiber element will be superposed on the patterns of all other elements, and the observed intensity distribution along the set of diffraction bands is the result of the simple addition of many separate effects arising from all fiber clements which partially intercept the beam of light in its passago from the source to the aperture of the eye. If the elements are very nearly uniform in diameter there will be a corresponding degree of coincidence of these patterns and, therefore, a maximum definition of the bands. Because of the variation in diameter, however, from fiber to fiber and along the length of each fiber, the elemental patterns are spread or contracted laterally in accordance with the variation in diameter. This effect tends to smooth out the distribution of light intensity and reduces the definition of the bands. The effect is particularly noticeable in the fusion of maxima and minima of higher orders (for $n=2,3$, etc.). The distribution of light in the band system, as observed for a bundle of fibers, thus bears a relation to the uniformity of the fibers as well as to their average diameter.

Corresponding to the case of a single fiber element, of a given diameter, we now take the position of the first minimum of intensity in the band system as a measure of the average diameter of the group of fibers. This presupposes, of course, by equation (1), that the observed position of the first minimum of intensity for the group of fibers is the harmonic mean of the positions for all elementary fiber lengths in the group. Denoting mean values by a bar over the corresponding letter, we have

$$
\bar{d}=\frac{C}{s_{1}}=\frac{C}{m} \underset{m}{\sum} \frac{1}{s_{1}, m}
$$

In this equation $m$ represents the number of $a$ fiber element and the summation is talien over the total observed length of fiber in the sample. This is the areraging process automatically performed by the instrment. Later tests will show how well the instrument fulfills its intended purpose.

It is shown by the preceding equations that the constant of the eriometer depends on the wave length, or wave-length composition, of the source of light. When white light is used in the illumination of the slit, the diffraction bands are, therefore, colored at the edgcs hecause of the small dispersion in the angle of diffraction corresponding to the finite ware-length range of the source. 'This effect does not interfere creatly with the use of the instrument when settings are male only on the first dark band. It does contribute, however, to the generial difinseness of the light distribution and to the uncertainty in settings of the criometer scale. A nonochromatic source of known ware length, if of sufficient intensity, would be an adrantage undoubtcdly and would rield an absolute measure of average diameter, for then the value of $C$ would be completely determined from the known construntion of the instrument.

In the present inicial study of the diffraction method unnecessary complications in the construction of the apparatus were avoided. come experiments were made with a red light fitter between the source and the slit of the instrument, but the resulting intensity of the difiracticn bands was not sufficient for precise or comfortable readings. suituble hed that a more intense source of white light combined with a suituble high-transmission red or green filter would be a distinct 
improvement and would serve all purposes as well as a more strictly monochromatic but more expensive source, such as the green spectral line of the mercury-vapor arc. When unfiltered white light is used, as with the present instrument, the choice of an effective average wave length for the source is somewhat arbitrary and uncertain. Hence, the constant $C$ is best determined for this instrument by measurements made on fine wires or fibers of uniform and known diameter.

The distance $D$ from the slit to the fibers is normally $30 \mathrm{~cm}$. For very fine fibers, however, the spread of the diffraction bands is so great that the first dark band may cover the greater part of the field of view; moreover, the intensity of the adjacent bright bands is much reduced under these circumstances so that it may prove very difficult to set precisely on the center of the first dark band. Hence, the observation tube is made in two parts (fig. 1), so that a length of $15 \mathrm{~cm}$. is readily removable. Then $D$ is reduced by one-half, giving $d=C / 2 s_{1}$.

\section{CALIBRATIOIN AND PERFORMANCE}

\section{CALIBRATION OF THE ERIOMETER SCALE}

To determine the constant of the eriometer and to obtain pertinent data on the performance of the instrument, comparative measurements were made with the eriometer and with a microscope on fine wires and various textile fibers. For these measurements samples were prepared by mounting 5 to 10 fibers, or wires, parallel to one another and streched taut across an aperture in the center of a cardboard form. (Fig. 1.) The ends of the fibers were held by soft wax. This cardboard form was properly mounted on the sample holder of the eriometer and secured in place by a little soft wax. These samples could be used with either instrument, inserted or removed as desired, without disturbing the arrangement of the fibers.

The microscope used in this work gave a magnification of approximately 100 diameters, and was equipped with a micrometer eyepiece. It may be recalled that the result obtained with the eriometer is the average diameter of such fiber lengths only which extend across the aperture of the eye. Hence, to obtain comparable data with the microscope, individual diameter measurements were made at intervals of $0.5 \mathrm{~mm}$. along the length of each textile fiber and covering a total length of approximately $5 \mathrm{~mm}$. The mean of the individual determinations for all the fibers was taken as the average diameter of the sample. Inasmuch as the wire samples are very uniform in diameter, the range of uncertainty in the average as determined with the microscope is estimated to be within a few tenths of a micron. These samples were also very carefully measured with the eriometer, several determinations of the mean displacement -1 being made at different times, each determination representing 10 settings of the eriometer scale. Two determinations (10 settings each) of each textile sample were made.

The mean of all determinations for each sample with the eriometer is represented by a single point in Figure 3 and there compared with the microscope average. The microscope averages, expressed in microns, are plotted against the reciprocal mean scale readings of the eriometer; that is, against the values of $1 / s_{1}$ in equation (2). The straight line passes through the origin of coordinates and its slope 
gives the required constant of the eriometer. After the constant was thus determined the micron scale for the eriometer was added to the chart.

With $d$ expressed in microns and $s_{1}$ in millimeters, the numerical value of $C$ is 167 . From the definition $C=\lambda D$, with $D=300 \mathrm{~mm}$, the effective wave length of the source is found to be $0.56 \mu$. This wave length falls, as it should, well within the region of high spectral luminosity of the source, and thus serves as a rough check on the correctness of the calibration.

In determining the slope of the calibration line consideration was given only to the wire samples and to two of the textile samples

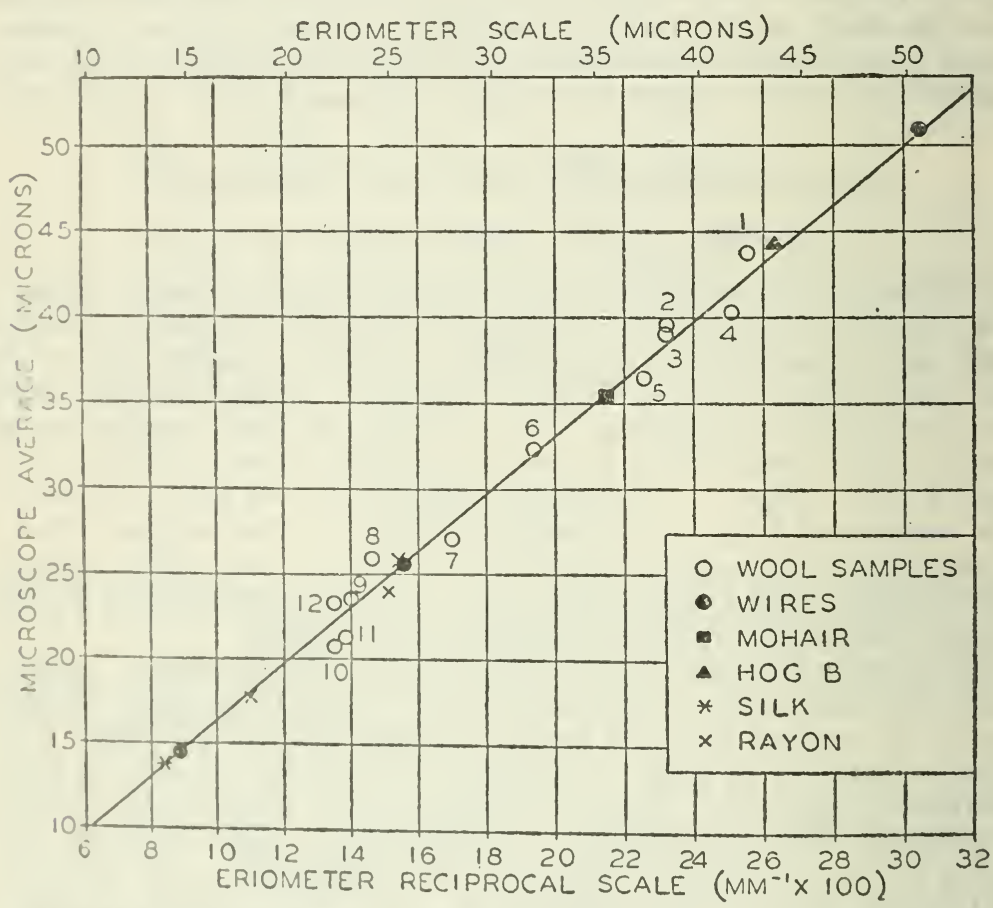

Fionr: 3.-Comparison of criometer readings with microscope averages

(mohair and hog bristles) which were shown by the microscope to be very uniform in diameter and moreover filled in an open range of diameters between the wire samples. A wide range of diameter rarintion within a sample introduces uncertainties in the microscope nrernere hecause of the limited number of individual diameter determinations made. Also the precision of the eriometer readings is slightly reduced under these conditions because of the poorer definition of the bands.

Each wool sample is composed of 10 fibers chosen at random from a sincle grade of the United States standards for wool top. One sample from ench of the 12 grades was mensured. These samples were chosen mainly for convenience because they provided wool-fiber groups of progressively varying average diameter. No attempt is thade in this paper to study the standards as such. The data on the 
wool samples were given no weight whatever in the determination of the constant of the eriometer. They will be used in later discussions of performance.

All measurements with both instruments on the textile fibers were made under constant atmospheric conditions of temperature and relative humidity in order to avoid any appreciable changes in diameter, particularly of wool, accompanying changes in moisture content of the material during the measurements. It is known that wool is extremely hygroscopic, and its diameter changes appreciably with moderate changes in moisture content.

\section{EFFECT OF IRREGULARITIES IN FORM AND ARRANGEMENT OF THE FIBERS}

In the development of the theory of the eriometer it was assumed that all fiber elements were parallel to the slit source of light. The diffraction phenomena were then discussed in their relation only to the diameter, or variation in diameter, of the fiber elements. The effect of the wave-length composition of the source, being constant, was of relatively small importance in the measurements, and only necessitated a calibration of the instrument by some independent method of observation. In the present section the theoretical discussion is extended to include the effect of varying orientation of the fiber elements from the previously assumed condition of parallelism.

In practical applications of the instrument it would be difficult and very inconvenient to fulfill closely this condition of parallelism within a large group of fibers. Some of the fibers may be in contact with adjacent fibers or crossing at one or more points; moreover, some of the finer grades of wool often exhibit a considerable degree of crimpiness in form so that the observed portion of the fiber length may be somewhat wave like in appearance unless particular care is taken to pull the fibers taut across the aperture in the sample holder; but if this operation is not performed with considerable care there is danger of stretching the fibers and thus appreciably reducing their normal cross section. It is therefore imperative to obtain some information on the effect of such irregularities in form and arrangement on the measurement of average diameter.

In the following theoretical discussion of the problem, the fiber elements of a group are assumed to be all the same in diameter, but may be oriented at varying angles with the slit source of light. In respect to the actual variation in diameter within a given group of fibers we may regard the uniform diameter assumed in the calculations as the average diameter of the group; then, providing there is no marked degree of correlation between the diameter of fiber elements and their orientation, the computations will give, at least approximately, the effect of orientation alone on the experimental determination of the average.

Let us consider first two perfectly straight fibers, or fiber elements, one of which is strictly parallel to the slit and the other inclined at an acute angle $a$. The diffraction bands are always arranged parallel to the slit, but exhibit their normal separation only along a line perpendicular to the axis of the fiber. Hence, as illustrated in Figure $4(A)$, the two sets of diffraction bands can not be coincident. The set corresponding to the fiber making an angle $a$ with the slit is displaced 
laterally along the line $O A$ by an amount $\Delta s$ relative to the normal set, the displacement being always toward the slit (negative direction with respect to $s$ ). If the rertical lines in the figure represent the centers of the dark bands, then

$$
-\Delta s_{1}=s_{1}(1-\cos a)
$$

The fractional error $e$ in the measurement of the diameter of the inclined fiber is obtained from equations (1) and (3): Thus

$$
e=\frac{\Delta d}{d}=-\frac{\Delta s_{1}}{s_{1}}=1-\cos a
$$

\section{(A)}
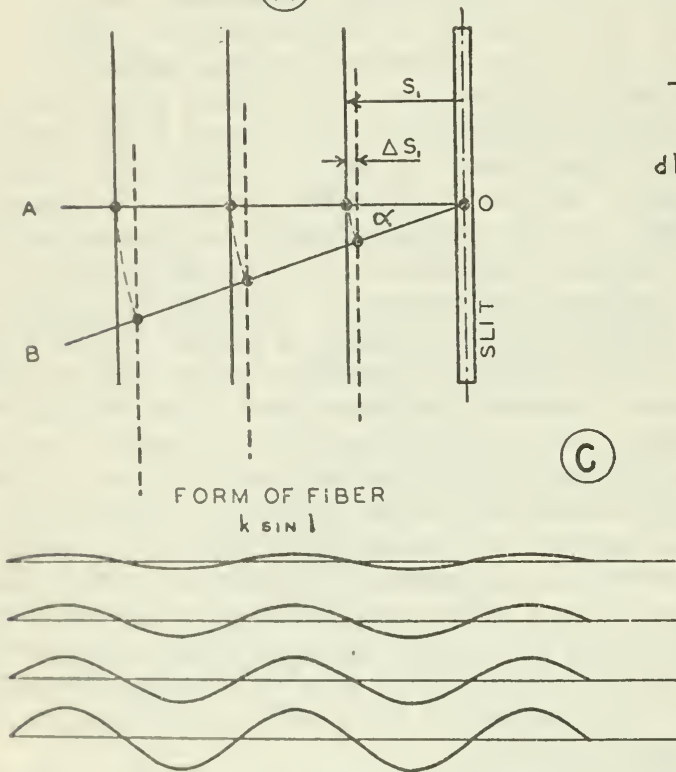

(C)

FIGCRE 4.-Fifect of irregularities in form and arrangement of the fibers

The error is an even function of $a$ so that plus and minus values of $a$ are equivalent. If the fiber makes an angle of either $\pm 12^{\circ}$ with the slit then the error in its measurement is +2 per cent. For angles of $\pm 5^{\circ}$ the corresponding error is about +1 per cent.

Equation (3) was verified experimentally for $a=12^{\circ}$ by carefully mounting four straight mohair fibers parallel to one another on a cardboard form and providing an arrangement whereby the fibers could be set cusily either parallel or at $12^{\circ}$ to the slit. A series of 60 scale settines was made with the fibers alternately parallel and inclined to the slit. The avernge scale reading for the inclined fibers was found to be 2 per cent lower than that for the parallel condition. The precision of a single determination with the eriometer is such that a lnrge number of successive scale settings are necessary to determine this difference. 
It is not very difficult to mount a bundle of straight fibers so that the condition of parallelism is fulfilled to a sufficient approximation to make the residual error negigibie.

Let us now calculate the error in the measurement of crimped fibers whose elements of length make continuously varying angles with the slit. For the purpose of calculation let the projection of each fiber be sinusoidal in form, as illustrated in Figure $4(C)$. A suitable variation in form, to simulate varying degrees of crimpiness, is obtained by a factor $k$. The form of the fiber is thus expressed by the function $k \sin l$.

The error in the separate measurement of any fiber element, if this measurement were possible, would be given by equation (4). We now require the average value $\bar{e}$ of this error function taken over a quarter period of the sinusoidal variation in form of the fiber. Referring again to Figure $4(A)$ we recall that $s_{1}$ is the position of the first minimum of intensity for a fiber element which is strictly parallel to the slit, and $\Delta s_{1}$ is the linear displacement of this minimum resulting from a given angular displacement of this element. The calculation of the average error for the quarter period involves the same assumptions which were made previously in the derivation of equation (2); and the total effect, for a group of fibers, is the result of the superposition of many separate effects arising from a large number of quarter periods, each of which is assumed to be of exactly the same form and, hence, acts in exactily the same way. We may regard the total number of quarter periods as so large that the effect of a fractional remnant may be disregarded. The error for the group of fibers is thus given by the error for a single quarter period.

A quarter period of variation in the form of the fiber is shown in Figure $4(B)$. The error $e$ due to any fiber element is properly weighted when multiplied by the element length $d l^{\prime}$. Let $d l=d l^{\prime}$ $\cos \alpha$ be the projection of $d l^{\prime}$ on a line parallel to the slit (axis for description of form of fiber). Then the resultant error $\bar{e}$, representing the joint effect of all fiber elements in the quarter period, is given ${ }^{14}$ by the equation

$$
\bar{e}=\frac{\int_{0}^{\pi / 2}(1-\cos \alpha) \sec \alpha d l}{\int_{0}^{\pi / 2} \sec \alpha d l}=1-\frac{\pi}{2 \int_{0}^{\pi / 2} \sec \alpha d l}
$$

From the relation $\tan \alpha=d(k \sin l) / d l=k \cos l$ we may express $\sec \alpha$ in terms of $l$, after which the equation readily reduces to the form

$$
\bar{e}=1-\frac{\pi}{2 \sqrt{1+k^{2}}} \cdot \frac{1}{E\left(\frac{\pi}{2}, K\right)}
$$

where $K=k / \sqrt{1+k^{2}}$, and $E\left(\frac{\pi}{2}, K\right)$ is the complete elliptic integral of

14 The average value of a function $f(x)$ with a set of weights $g(x)$ is the ratio of the integral of $f(x) g(x)$ to the integral of $g(x)$, the integrations being taken over the range of $x$ for which the average of $f(x)$ is
required. 
the second lind. The value of this integral, for various values of $K$, may be read directly from tables ${ }^{15}$ of the elliptic functions.

In this manner the numerical data in Figure $4(C)$ were obtained. Each datum is the theoretical percentage error in a diameter measurement with the eriometer for a single fiber, or for a group of fibers, when each fiber of the group has the corresponding form $(k \sin l$, with $k$ constant) depicted in the figure. It may be noted that no particular length unit is involved in the mathematical description of these forms; hence, any form may be uniformly contracted or expanded at will so that any desired number of quarter periods may be included within the fixed aperture area of the eriometer. In this way a given form may be made to match as closely as possible the actual projected configuration of a given fiber. Furthermore, the degree of contraction or expansion may be varied at will to match different fibers in a given sample or different portions of the same fiber. The only requirement in this respect which has been imposed on the above calculations is that the contraction or expansion shall be uniform in all directions in the plane of the aperture so that the geometrical form of the theoretical fiber is not changed. If different forms (different values of $k$, or different functional forms) are used to represent different members of a given group of fibers, then the resultant crror would be found as a mean value of the properly weighted theoretical errors applying to the different forms used.

The preceding computations show that the resultant effect of all irregularities in the orientation of the fibers is a contraction of the band system toward the central bright slit; so that the error is always in the same sense, giving values which are always too large.

Several experiments were performed in an attempt to obtain a partial check on the magnitude of these theoretical errors. In each of these experiments four characteristically crimped fibers were selected from one of the finer grades of wool top and mounted with wax on a cardboard form. The average diameter was determined with high precision by making a large number of settings of the eriometer scale. The sample holder was then removed from the instrument and the fibers straightened approximately, taking care not to stretch the fibers appreciably during this operation. The measurement of a vernge dinmeter was then repeated and the percentage difference computed. The results all showed a definite decrease in the value of the average diameter measured after the fibers had been struightened. This decrense varied from 5 per cent to nearly zero, however, even though the irregularities in the fibers, as originally mounted, appenred very much the same in each experiment.

In consideration of these results it should be borne in mind that the straightening operation may involve some rotation and untwisting of fiters with noncircular cross section so that the average projected dimneter might be altered in this process. Furthermore, it is probable that the tendency toward crimpiness is greatest for fiber lengths of smallest dinmeter; this tendency would off-set the orientation crror, so that the measured effect would be less than that calculated on the assmuption that there was no correlation between diameter and orientation of fiber elements. Again, it is evident that no set of crimped fibers wil! actually assume such a regular sinusoidal varia-

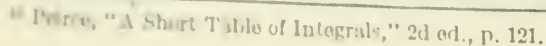


tion in form as depicted in Figure $4(C)$. Some of the mounted fibers may be quite wave like in appearance over a small portion of their total length, but twisted in an adjacent portion so that their projection in the line of sight is straight. This latter portion of the total fiber length would contribute no appreciable error due to its form. On the whole, judging from a visual comparison of irregularities in the mounted fibers with the theoretical forms shown in Figure $4(C)$, it appears that the theoretical errors are somewhat too large so that the effect may be exaggerated in the theoretical discussion. It is difficult to obtain an adequate experimental confirmation of the theory. In 6 successive mountings of badly crimped fibers the average experimental error was only 3 per cent. It would not be difficult to straighten such fibers approximately so that the residual experimental error in their measurement would be insignificant.

In regard to overlapping and crossing of the fibers it is evident that these irregularities should be avoided as much as possible. Although the effect at the intersection of two crossed fibers is negligible, the crossing in itself implies a varying orientation. Overlapping of two approximately parallel fibers is equivalent to one very much wider fiber and, hence, must increase the observed average. Some experiments were performed in which it was attempted to mount two uniform mohair fibers side by side in close contact but not overlapping, so that the instrument would indicate the combined thickness of the two fibers. It was found, however, that the contact was never close enough to effect the looked-for change in the separation of the bands. Apparently there is no danger of any appreciable error from this cause in the measurement of wool or other textile fibers.

From the results of these studies of irregularities in orientation it is advisable to use reasonable care in the arrangement of the fibers so that they lie approximately parallel to one another with a minimum of crossing and overlapping. If the fibers show a high degree of crimpiness in form, some error in the measurement may be expected, al though experiment has shown that the effect is not serious. It is doubtful, in view of later considerations of diameter distribution and accuracy requirements, if errors arising from casual irregularities in mounting can be regarded as a serious disadvantage of the method.

\section{DISPERSION OF DIAMETERS IN WOOL SAMPLES}

An analysis of data obtained on the wool samples will illustrate the wide range and varied distribution of diameters, which are averaged in a single determination with the eriometer.

Each wool sample consisted of 10 fibers carefully mounted parallel to one another. The width of each bundle was from 4 to $5 \mathrm{~mm}$, and 10 individual diameter measurements with the microscope were made on each fiber at intervals of $0.5 \mathrm{~mm}$ along its length. Thus 100 individual diameter measurements were made on each sample, covering an area approximately $5 \mathrm{~mm}$ square. This area fully covered the aperture of the eye when the bundle was measured with the eriometer.

The diameter-frequency distribution in each wool sample is represented by one of the curves in Figure 5 . In this figure the abscissae represent percentage deviations from the mean of 100 diameters. The computed deviations have been grouped into classes, each class $43324^{\circ}-31-14$ 
covering a range of 6 per cent. The number of cases falling within a given class is represented by an ordinate erected on the mid-class value. The points thus determined are connected by straight lines forming a frequency polygon, or roughly, a frequency curve.

It may be emphasized that these frequency curves do not represent directly the variation in diameter between different fibers of a group; they are intended to illustrate only the range and distribution of diameters among fiber elements as actually presented to the instrument for averaging. The diameter variation along the length of the fiber's is often less than the variation between fibers, so that a sample containing a few fibers of approximately the same diameter, but quite

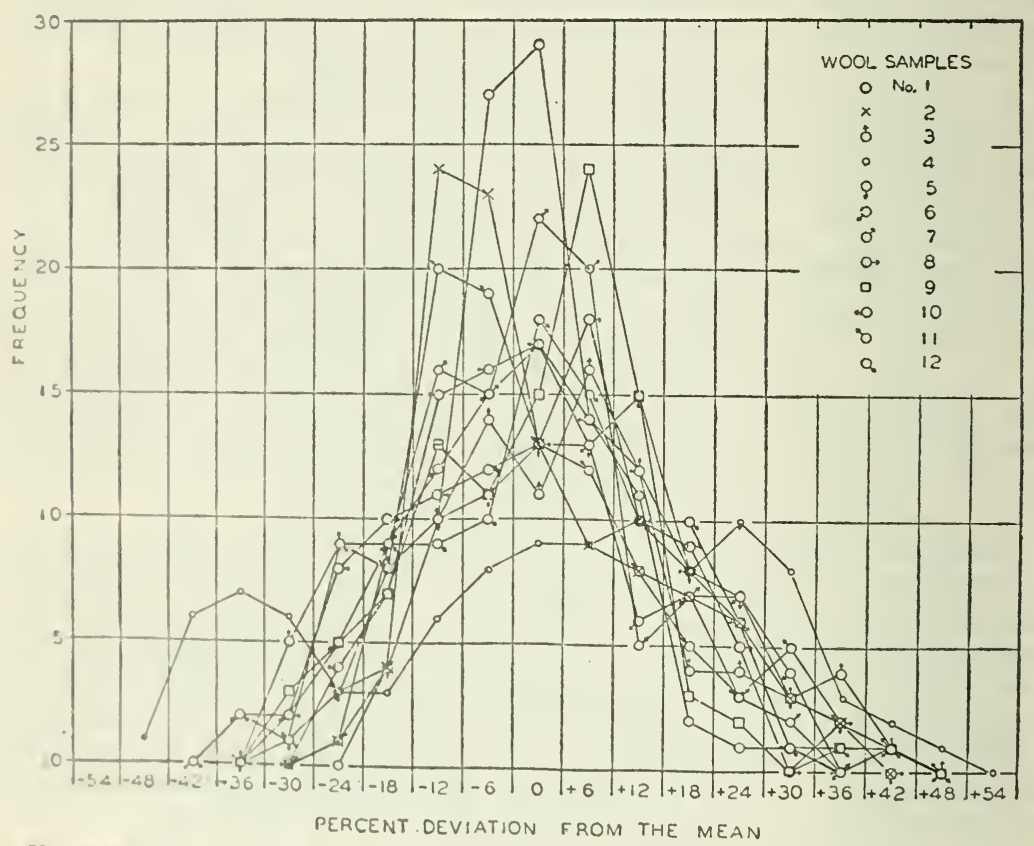

Figure: 5.-Diameter-frequency distribution in wool samples (100 observations on cach sample; class interval 6 per cent)

different from the svernge diameter of the group, would show a definite maximum at a corresponding distance from the center of the diagram. The mmber of fibers in the sample measured is too small to be fully represcutative of the total population of fibers in the larger sample from which the fibers were taken. These considerations explain some of the ofserved irregularities in diameter distribution as exemplified, for unstance, by sample No. 4; but it is undoubtedly quite possible to in lange commercial sas shown representing true diameter distributions

t coull tearer of samples.

these dinmeter of stiewness may be noted in the general trend of cocptionally lare diaureters showing a tendency in these samples for oppesite extreme The frequently than the the majority of in uverage diameter. 
If the diameters of the fiber elements are grouped very closely about their average value then the sets of diffraction bands corresponding; respectively, to individual elements are also all nearly coincident and a maximum definition of the observed bands is attained. With textile fibers, however, there is always a considerable dispersion of diameters which tends to smooth out the maxima and minima of light distribution and thereby decrease the definition of the bands. This effect is therefore a definite indication of the dispersion of diameters in the sample. In Section. IV it will be indicated how a quartitative measure of the dispersion might be obtained. As the range of diameters increases, and particularly, if the distribution curve is broad and flat, settings of the eriometer scale become increasingly difficult and uncertain. The instrument is thus imited in its range of application. Not every possible diameter distribution may be averaged with the instrument. Definite reliable settings were readily obtained, however, with all the wool samples whose diameter distribution curves are given in Figure 5.

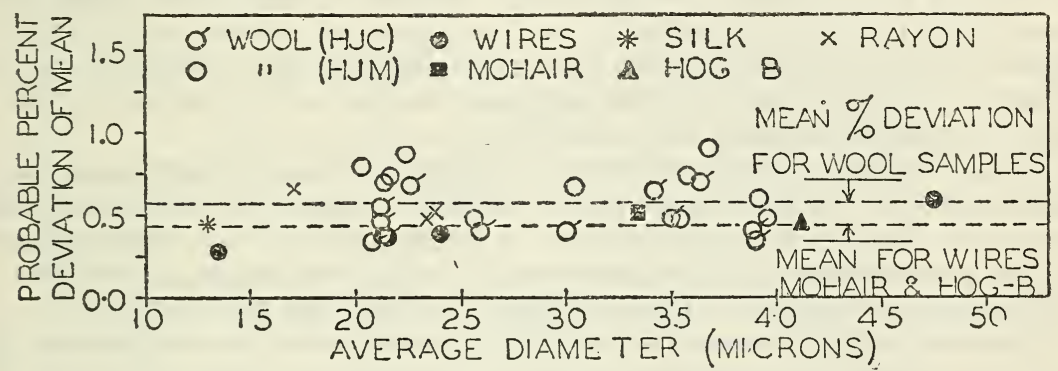

FIgURE 6.-Precision of measurements with the eriometer

\section{PRECISION AND ACCURACY OF IMGASUREMENTS}

In the following discussion of the precision of measurements a single standard determination of average diameter with the eriometer is defined as the result derived by equation (2) when $\bar{s}_{1}$ is the mean of 10 consecutive settings of the eriometer scale. For the purpose of expressing the precision, the probable percentage deviation ${ }^{16}$ of this mean scale setting was computed for each determination of average diameter. By the relation $\Delta s / s=-\Delta d / d$ (equation (4)), these deviations are also the probable percentage deviations of single standard aeterminations of average diameter.

The data are given in Figure 6. It is shown that there is no consistent change in the precision of measurements over the entire range of average diameters covered by these observations; furthermore, there is only a small decrease in precision, as the dispersion of diameters in the sample increases markedly in passing from the very uniform wire and textile samples to the most nonuniform samples of wool. The probable percentage deviation of a single standard determination is, roughly, 0.5 per cent for the wool samples and decreases only slightly for the wires and other textile samples of nearly uniform diameter. Assuming no constant sources of error in the method or

16 Probable percentage deviation of the mean of 10 observations is given by the product $0.0282 \Sigma$ (resid. uals). Merriman "Method of Least Squares" 8 th ed., p. 93 and Table 4. (Short formula.) 
instrument, and the normal law for symmetrical distribution of successive scale settings about the mean, these data on precision may be interpreted as follows: In a single standard determination of the average diameter of a wool sample the chances are even that the observed value will be within \pm 0.5 per cent of the true value, and it is almost certain that the observed value will not differ from the true ralue by more than \pm 2 per cent.

We may recall at this point that each value given (fig. 3 ) for the average diameter of a wool sample, as measured with the eriometer, is the mean of two determinations, each by a different observer. It is of interest to note that no consistent difference was found between the two sets of data, corresponding, respectively, to the two different observers. The average difference, taken without regard to sign, between corresponding values for the two observers was 1.2 per cent. It appears, therefore, that there is no considerable personal fuctor in these measurements. One may expect some consistent variation between the average scale settings of individual members of a group of observers, because of the colored edges of the diffraction bands; the criterion for settings on the center of a band may depend somewhat on the color-vision characteristics of the observer. This effect is probably small, however, and would be eliminated by the use of a monochromatic source of light.

When dealing with broad irregular diameter distributions one may well question the adequacy of the simple averaging process of the eriometer, as previously explained in Section II. Some information on the performance of the instrument in this respect is gained from the diameter analysis of the wool samples with the microscope.

'The wool samples were all carefully mounted so that no appreciable errors in their measurement with the eriometer could result from residual irregularities in orientation. Assuming no significant errors in the individual diameter determinations with the microscope, and that a truly representative average is obtained from the 100 individual determinations on each sample, then the accuracy of the averaging process of the eriometer may be adjudged by the grouping of points for the wool samples about the calibration line in Figure 3 .

We find here no consistent differences between the averages obtained with the eriometer and the microscope. Sample numbers are attached to the points in Figure 3 and to corresponding distribution curves in Figure 5. By comparison, for a given sample, of the departure from the calibration line with the spread or flatness of the corresponding distribution curve, we see that these departures are not definitely correlnted with the character of the diameter distribution. Thus, the arerage diameter of samples 4,3 , and 12 , which show the brondest and flattest distribution curves, do not depart further or consistently in direction from the calibration line than samples 1, 7, or 2, which show the greatest concentration of diameters nbout the mean. Samples 11 and 2, with the same type of distribution curve, full on opposite sides of the calibration line. Such variations as found between results with the two instruments are not inconsistent with the precision of the eriometer (fig. 6) and with the dispersion of diameters in the samples.

The avernge dinmeter measured with the criometer compares very favorably with the microscope average. The differences observed are not grater than $2 \mu$. Although the microscope is undoubtedly 
the more accurate for nearly uniform samples, such as the wires, it is probable that in the case of the nonuniform textile fibers the eriometer yields an average value which is more accurate than the microscope average, for 10 individual determinations over a $5 \mathrm{~mm}$ length of a nonuniform fiber may not adequately gage the fiber.

\section{DISCUSSION AND CONCLUSIONS}

The most commendable feature of the diffraction method is its ability to combine into a single averaged result the effect of a wide range of projected diameters as distributed in a group of fibers (or particles). For many purposes this gives the instrument unquestionable advantage over every form of micrometer which measures only a single interval at once. An average obtained in a few minutes with the eriometer may require an hour or more to duplicate with a microscope; moreover, the method averages the continuous diameter variation along the entire length of fiber under observation, and not, as in any microscopic method, the diameters at a limited number of points.

It should be emphasized, however, as previously noted, that the method is not suitable for averaging all possible diameter distributions. It is inherent in the method that the range of diameters must not be too large combined with a frequency distribution curve which is fiat or very irregular. For best results there should be a pronounced concentration of diameters about a single class.

Off-setting this limitation it may be remarked that whenever the method does fail to function, any practical significance which may be attached to the average diameter must be small. In an application of the eriometer to the grading of wools a sample which is not sufficiently uniform in diameter to permit a reading to be obtained with the instrument is also, by the same token, not representative of any one class of wools or even a narrow range of classes.

Aside from the somewhat uncertain effect of gross irregularities in the mounting of the fibers, it is believed that the precision and accuracy attained in the measurements are sufficient for practical applications of the method. When one considers that the diameters in a sample of wool may vary over a range of 100 per cent, and may be distributed within this range in a variety of different ways, it would seem that higher degrees of precision and accuracy in the measurement of the average could have no particular significance from the point of view of the utility of the material for any manufacturing purpose. It is, perhaps, only in a desire to distinguish sharply between limits arbitrarily set for different grades that greater refinement in the measurements may be considered desirable.

By the use of a monochromatic source of light, or a narrow spectral range in filtered white light, a definite characteristic wave length may be assigned to the source and an absolute measurement thereby obtained. Thus the eriometer is readily made independent of calibration in terms of another instrument.

The most serious disadvantage of the present instrument is its inability to cover a larger area of the sample, or greater total length and number of fibers, in a singie determination. A few fibers chosen at random from a large sample may exhibit a very erratic diameter distribution which is not truly characteristic of the material; and if the 
fibers are not straight and approximately circular in cross section it is conceivable that the distribution or average of projected diameters may depend in this case to a small degree on the mounting of the fibers. It would obviously be desirable if measurements could be made directly on a more fully representative sample of the material.

As stated in the introduction, it is also desirable that the determination of the average be supplemented by a suitable measure of variation. It has been shown how a qualitative indication of variation is provided in the present instrument by the appearance of the difiraction bands. We recall that the contrast between the maxima and minima of intensity distribution decreases with increased dispersion of diameters in the sample. It is possible that a measurement of the intensity distribution along the system of diffraction bands would vield pertinent information and quantitative expression of the essential features of the actual frequency distribution of diameters in the sample. The sample could be arranged so that equal lengths of individual fibers are always included in the measurements, and the results could then be interpreted as a measure of the variation between fibers rather than between fiber elements as depicted in Figure 5. The intensity distribution curve would also provide for greater precision in the determination of the position of minimum or maximum intensity and the average diameter derived therefrom.

These desirabie features of the diffraction method could readily be incorporated in a more complex and costly construction of the instrument. To cover a larger area of the sample we may replace the lens of the eye, with its small aperture, by a lens of any desired aperture over which the parallel bundle of fibers could be spread and mounted, as before. The diffracted light may then be brought to a focus on a screen and the diffraction pattern viewed directly; or better, the pattern may bo formed in the focal plane of a suitable eyepiece. Various devices may then be employed to obtain a measure of the angular separation of the bands. Some micrometer arrangement is necessary either to shift the bands across a fixed mark in the field of view, or to move a pointer across the stationary system of bands. If the intensity distribution is to be obtained, the eyepiece may be replaced by a suitable form of radiometer, employing either visual, photo-electric, or thermoclectric methods for measuring and recording relative variations in intensity.

From the results of the present investigation it is believed that the difraction method offers considerable opportunity for the further devoloment of instruments which would prove very useful in the textile field. Such instruments, however, would require a more bulky, delicite, and costly construction than the present instrument, and may require more care and skill in their operation. The instrument herein described will serve many purposes for which only a direct average diameter is required for a small group of fibers. Extensions to larger samples may be made if desired by averaging many small specimens properly sclected from the larger sample. The simplicity of construction and operation and its portability commend this uppliment not only for use in the laboratory, but also for extended

Washington, January 26, 1931. 